\title{
LIMIT FORMULAE AND JUMP RELATIONS OF POTENTIAL THEORY IN SOBOLEV SPACES
}

\author{
MARTIN GROTHAUS AND THOMAS RASKOP
}

\begin{abstract}
In this article we combine the modern theory of Sobolev spaces with the classical theory of limit formulae and jump relations of potential theory. Also other authors proved the convergence in Lebesgue spaces on $\partial \Sigma$ for integrable functions, see for example [7] or [9]. The achievement of this paper is the $L^{2}(\partial \Sigma)$ convergence for the weak derivatives of higher orders. Also the layer functions $F$ are elements of Sobolev spaces and $\partial \Sigma$ is a two dimensional suitable smooth submanifold in $\mathbb{R}^{3}$, called regular $C^{m, \alpha}$-surface. We are considering the potential of the single layer, the potential of the double layer as well as their first order normal derivatives. Main tool is the convergence in $C^{m}(\partial \Sigma)$ which is proved with help of some results taken from [14]. Additionally, we need a result about the limit formulae in $L^{2}(\partial \Sigma)$, which can be found in [16], and a reduction result which we took from [19]. Moreover we prove the convergence in the Hölder spaces $C^{m, \beta}(\partial \Sigma)$. Finally, we give an application of the limit formulae and jump relations to Geomathematics. We generalize a density results, see e.g. [11], from $L^{2}(\partial \Sigma)$ to $H^{m, 2}(\partial \Sigma)$. For it we prove the limit formula for $U_{1}$ in $\left(H^{m, 2}(\partial \Sigma)\right)^{\prime}$ also.
\end{abstract}

Key words and phrases: Limit formulae, Jump relations, Sobolev spaces, Submanifolds, Potential theory.

2000 Mathematics Subject Classification: 31B25, 31B10, 32C25, $46 \mathrm{E} 35$.

\section{INTRODUCTION}

In this article we prove the convergence of the limit formulae and jump relations of potential theory in several norms. More precisely, we investigate the potential of the single layer $U_{1}$ and the potential of the double layer $U_{2}$, defined by

$$
\begin{aligned}
U_{1}[F](x) & :=\int_{\partial \Sigma} F(y) \frac{1}{|x-y|} d H^{2}(y), \\
U_{2}[F](x) & :=\int_{\partial \Sigma} F(y) \frac{\partial}{\partial \nu(y)} \frac{1}{|x-y|} d H^{2}(y),
\end{aligned}
$$

for all $x \in \mathbb{R}^{3} \backslash \partial \Sigma$, where $\partial \Sigma$ is a regular surface in $\mathbb{R}^{3}$ and $F$ is a given function on $\partial \Sigma$, called layer function. Furthermore, we investigate the first order normal derivatives of $U_{1}$ and $U_{2}$. A regular surface $\partial \Sigma$ divides $\mathbb{R}^{3}$ into a bounded domain $D$ and an unbounded domain $\mathbb{R}^{3} \backslash \bar{D}=\Sigma$. The limit formulae describe the behavior of the potentials when approaching the surface $\partial \Sigma$. In general, the limits are different when approaching either from the inner or the outer space. These circumstance is called jump relation and can be obtained by the results for the limit formulae. Therefore we restrict ourselves to the limit formulae, while each jump relation can be computed easily by taking either 
the difference or the sum of the corresponding limit formula. Note that $U_{1}$ and $U_{2}$ are analytic, harmonic functions as well in the inner as in the outer space, even for regular $C^{2}$-surfaces $\partial \Sigma$ and integrable $F$. Nevertheless we need additional regularity assumptions in order to prove that the limits, when approaching to the surface, exist. We need the potentials to be Hölder continuous, then we know that a unique Hölder continuous continuation onto $\partial \Sigma$ exists. If we want to prove the convergence in $C^{m}(\partial \Sigma)$ norm, also the derivatives have to be Hölder continuous. These properties can be ensured for sufficiently smooth surface $\partial \Sigma$ and $F$. From literature we know that for regular $C^{2}$ surfaces $\partial \Sigma$ and continuous $F$ we have

$$
\begin{aligned}
\lim _{\tau \rightarrow 0^{+}} U_{1}[F](x \pm \tau \nu(x)) & =U_{1}[F](x) \quad \text { for all } x \in \partial \Sigma \\
\lim _{\tau \rightarrow 0^{+}} \frac{\partial U_{1}}{\partial \nu}[F](x \pm \tau \nu(x)) & =\frac{\partial U_{1}}{\partial \nu}[F](x) \mp 2 \pi F(x) \quad \text { for all } x \in \partial \Sigma, \\
\lim _{\tau \rightarrow 0^{+}} U_{2}[F](x \pm \tau \nu(x)) & =U_{2}[F](x) \pm 2 \pi F(x) \quad \text { for all } x \in \partial \Sigma, \\
\lim _{\tau \rightarrow 0^{+}} \frac{\partial U_{2}}{\partial \nu}[F](x \pm \tau \nu(x)) & =\frac{\partial U_{2}}{\partial \nu}[F](x) \quad \text { for all } x \in \partial \Sigma .
\end{aligned}
$$

even uniformly in $x \in \partial \Sigma$. The first three formulae can be found in [15], while we get the last formula, using results from [4] and [24]. In this case we have to assume $F \in C^{1, \alpha}(\partial \Sigma)$. We can even prove that this convergence holds in $C^{0, \beta}(\partial \Sigma)$-norm, provided the additional assumptions

$$
\begin{array}{ll}
F \in C^{0, \alpha}(\partial \Sigma) & \text { for formula } 1, \\
F \in C^{0, \alpha}(\partial \Sigma) & \text { for formulae } 2 \text { and } 3,
\end{array}
$$

are fulfilled, where $0 \leq \beta<\alpha \leq 1$. Moreover, we are able to prove that these formulae stay valid in $C^{m}(\partial \Sigma)$-norm for a regular $C^{m+1, \alpha}$-surface $\partial \Sigma$ and in $C^{m, \beta}(\partial \Sigma)$-norm for an regular $C^{m+2}$-surface $\partial \Sigma$, if

$$
\begin{array}{cl}
F \in C^{m-1, \alpha}(\partial \Sigma) & \text { for formula } 1, \\
F \in C^{m, \alpha}(\partial \Sigma) & \text { for formulae } 2 \text { and } 3, \\
F \in C^{m+1, \alpha}(\partial \Sigma) & \text { for formula } 4,
\end{array}
$$

where $m \in \mathbb{N}$ and $0 \leq \beta<\alpha \leq 1$. In the proofs we mainly use results, taken from [14]. The convergence in $C^{m}(\partial \Sigma)$-norm is basic for the convergence in the Sobolev spaces $H^{m, 2}(\partial \Sigma)$. Another result, which is essential in the proof of our main result is the convergence of $U_{1}, U_{2}$ in $L^{2}(\partial \Sigma)$-norm, proved in [16]. We have this convergence for each regular $C^{2}$-surface $\partial \Sigma$ and each $F \in L^{2}(\partial \Sigma)$. The main result of the article is the following. We prove the convergence of the formulae above in $H^{m, 2}(\partial \Sigma)$ under the conditions

$F \in H^{m, 2}(\partial \Sigma)$ and $\partial \Sigma$ a regular $C^{m+1, \alpha}$-surface for formula 1, $F \in H^{m+1,2}(\partial \Sigma)$ and $\partial \Sigma$ a regular $C^{m+2}$-surface for formulae 2 and 3 , $F \in H^{m+2,2}(\partial \Sigma)$ and $\partial \Sigma$ a regular $C^{m+3}$-surface for formula 4 .

For it we use the BLT Theorem, which gives an unique extension of the potential operators from $C^{m}(\partial \Sigma)$ to $H^{m, 2}(\partial \Sigma)$, provided we are able to prove that these operators are 
continuous in $H^{m, 2}(\partial \Sigma)$-norm on that dense subset. Therefore we estimate the potential operators on parallel surfaces $\partial \Sigma^{ \pm \tau}$ by a constant independent of $\tau>0$. The advantage is that we avoid the singular integrals in the case when $x$ is itself an element of the surface. But the problem is to get a constant independent of $\tau>0$. To get this, we use the mappings from the surface to $\mathbb{R}^{2} \times\{0\}$, translate differentiation with respect to $x$ to differentiation with respect to $y$ and apply integration by parts to transfer the differential operators to the layer function $F$. Furthermore, we have to use a reduction result derived from the transformation formula for the Laplace operator. This helps us to get rid of higher order derivatives in direction $y_{3}$ which can not be treated with help of integration by parts, because we are only integrating over the variables $y_{1}$ and $y_{2}$. In the last section we prove that the results are applicable to Geomathematics. We prove that the system of mass point representations as well as the systems of inner and outer harmonics are dense in $H^{m, 2}(\partial \Sigma)$ for arbitrary $m \in \mathbb{N}$. With help of the proved results we are able to define $U_{1}^{ \pm \tau}[F]$ for each $F \in\left(H^{m, 2}(\partial \Sigma)\right)^{\prime}$ and $\tau \in\left[0, \tau_{0}\right]$, where $\partial \Sigma$ is a regular $C^{m+2}$-surface. Moreover we are able to prove that the limit formula for $U_{1}$ even holds in this abstract setting. This enables us to extend the result from [11], about the density of the function systems in $L^{2}(\partial \Sigma)$, to $H^{m, 2}(\partial \Sigma)$.

The article is organized as follows. In Section 2 we give the definitions of the surfaces and function spaces we use, as well as some properties of them. Furthermore, we formulate results which are important tools in the proofs of the main results and we define the layer potentials as well as their first order normal derivatives. These are subject of our investigation in the following sections. Also some basic properties are stated. In Section 3 we state the limit formulae of potential theory for the first time, pointwisely and uniformly, i.e., in $C^{0}(\partial \Sigma)$-norm. These results are the basis of our further considerations. They are mainly taken from literature, except of the limit formula for $\frac{\partial U_{2}}{\partial \nu}$. In Section 4 we present the first of our three main results. This is the convergence of the limit formulae in $C^{m}(\partial \Sigma)$-norm. The second one follows in Section 5 as the extension to the convergence even in the Hölder spaces $C^{m, \beta}(\partial \Sigma)$. Section 6 is essential for the proof of the convergence in $H^{m, 2}(\partial \Sigma)$-norm, which is proved in Section 7. It contains the convergence in $L^{2}(\partial \Sigma)$, which is published in [8] as well as in [16] and has its origin in [12]. Moreover, an important result about the continuity of the potential operators with respect to the $L^{2}(\Sigma)$-norm is stated and proved. Finally we prove the density of several function systems from Geomathematics in $H^{m, 2}(\partial \Sigma)$, as well as the limit formula of $U_{1}$ for $F \in\left(H^{m, 2}(\partial \Sigma)\right)^{\prime}$ in Section 8 .

The main progress achieved by this article can be summarized by the following core results:

- The convergence of the limit formula for $U_{1}, U_{2}, \frac{\partial U_{1}}{\partial \nu}$ and $\frac{\partial U_{2}}{\partial \nu}$ in $C^{m}(\partial \Sigma)$-norm, $m \in \mathbb{N}$ is proved.

- Moreover this convergence is shown to be even a convergence in $C^{m, \beta}(\partial \Sigma)$-norm, $m \in \mathbb{N}_{0}:=\mathbb{N} \cup\{0\}$ and $0<\beta<1$.

- Finally, the convergence in $H^{m, 2}(\partial \Sigma)$ is proved. Moreover, the convergence of the limit formula for $\frac{\partial U_{2}}{\partial \nu}$ in $L^{2}(\partial \Sigma)$-norm is shown. 
- An application of the limit formulae to Geomathematics is given. We prove that the systems of mass distributions as well as the systems of inner and outer harmonics, which are known to be dense in $L^{2}(\partial \Sigma)$, are even dense in $H^{m, 2}(\partial \Sigma)$, $m \in \mathbb{N}$, for a regular $C^{2 m-1,1}$-surface $\partial \Sigma$. Moreover, we extend $U_{1}$ to an operator acting on $\left(H^{m, 2}(\partial \Sigma)\right)^{\prime}$ and we prove that the limit formula for $U_{1}$ holds in this very general setting.

Martin Grothaus and Thomas Raskop, Mathematics Department,

University of Kaiserslautern, P.O.Box 3049, 67653 Kaiserslautern, Germany.

Email: grothaus@mathematik.uni-kl.de 